\title{
ON A TWO-PARAMETER FAMILY OF NONHOMOGENEOUS MEAN VALUES
}

\author{
FENG QI
}

\begin{abstract}
In the article, a two-parameter family of nonhomogeneous means is considered, and its basic properties and monotonicity are investigated. This paper is dedicated to my advisor, Prof. Yi-Pei Chen, at Ximaen University.
\end{abstract}

\section{Introduction}

The history of mean values is long. One finds the history in $[2,7]$. A survey of some recent developments can be found in $[8,12,13]$. The mean values are related to the Mean Value Theorem for derivative and for integral, which is the bridge between the local and global properties of functions. Inequalities of mean values are the main part of theory of analytic inequalities, they have explicit geometric meanings [16].

The simplest and classical means are the arithmetic mean or average, $A(x, y)=$ $(x+y) / 2$, the geometric mean or mean proportional, $G(x, y)=\sqrt{x y}$, and the harmonic mean, $H=G^{2} / A$. They have been generalised, extended, variegated, and refined to a lot of forms. The root-mean-square is defined as $N=(G+A) / 2$ and the power means or Hölder means as $M_{r}(x, y)=\left(\left(x^{r}+y^{r}\right) / 2\right)^{1 / r}, r \neq 0, M_{0}(x, y)=G(x, y)$. In this paper, the variables $x$ and $y$ are positive.

Further evolution led to multivariable means with $\left(x_{1}, x_{2}, \ldots, x_{n}\right)$ replacing $(x, y)$, to abstracted means $M_{\varphi}=\varphi^{-1}((\varphi(x)+\varphi(y)) / 2)$ which reduce to $M_{r}$ when $\varphi(x)=x^{r}$, to weighted means which are given by $(1-\alpha) x+\alpha y$ and $x^{1-\alpha} y^{\alpha}, 0 \leq \alpha \leq 1$, to Lehmer means $L_{p}(x, y)=\left(x^{p}+y^{p}\right) /\left(x^{p-1}+y^{p-1}\right), p>0$, which reduce to anti-harmonic mean $L_{2}(x, y)=\left(x^{2}+y^{2}\right) /(x+y)$.

Along with means $M_{r}$ there are more extended means of particular interest. Pólya and Szegö in [15] defined the logarithmic mean $L$ by

$$
L=L(x, y)=(x-y) /(\ln x-\ln y)
$$

for $x>0, y>0$ and $x \neq y$, and $L(x, x)=x$.

Received June 20, 1997; revised October 10, 1997.

1991 Mathematics Subject Classification. Primary 26A48, Secondary 26D10.

Key words and phrases. Two-parameter family, nonhomogeneous means, basic property, monotonicity, Tchebycheff integral inequality.

The author was partially supported by NSF grant no. 974050400 of Henan Provnice, The People's Republic of China 
Galvani in [6] considered the extended logarithmic means or Stolarsky's means:

$$
S_{p}(x, y)=\left(\frac{y^{p}-x^{p}}{p(y-x)}\right)^{1 /(p-1)}, \quad x \neq y, p \neq 0,1 ;
$$

and $S_{p}(x, x)=x$; which is reduced to $S_{0}(x, y)=L(x, y)$, and to the identric mean of the exponential mean $I(x, y)$ :

$$
S_{1}(x, y)=I(x, y)=e^{-1}\left(x^{x} / y^{y}\right)^{1 /(x-y)}, \quad x \neq y ;
$$

and $S_{1}(x, x)=I(x, x)=x$.

They symmetric means $Q_{p}(x, y)$ is also defined by

$$
Q_{p}(x, y)=\left(x^{r} y^{s}+x^{s} y^{r}\right) / 2
$$

where $r=(1+\sqrt{p}) / 2, s=(1-\sqrt{p}) / 2, p \geq 0$.

Ren-er Yang and Dong-ji Cao in [24] and Horst Alzer in [1] generalized $L(x, y)$ to the one-parameter means:

$$
\begin{aligned}
& J_{p}(x, y)=\frac{p\left(y^{p+1}-x^{p+1}\right)}{(p+1)\left(y^{p}-x^{p}\right)}, \quad x \neq y, p \neq 0,-1 \\
& J_{0}(x, y)=L(x, y), \quad J_{-1}(x, y)=G^{2} / L \\
& J_{p}(x, x)=x
\end{aligned}
$$

Here, $J_{1 / 2}(x, y)=h(x, y)$ is called Heron mean and $J_{2}(x, y)$ the centroidal mean.

Ji Chen and Hai-bing Shu [5] introduced the extended Heron means $h_{n}(x, y)$ by

$$
h_{n}(x, y)=\frac{1}{n+1} \sum_{k=0}^{n} x^{1-k / n} y^{k / n}
$$

and they verified that $h_{n}$ is a decreasing sequence.

Stolarsky in [20] defined a two-parameter family of extended means $E(r, s ; x, y)$ :

$$
\begin{aligned}
& E(r, s ; x, y)=\left(\frac{r}{s} \cdot \frac{y^{s}-x^{s}}{y^{r}-x^{r}}\right)^{1 /(s-r)}, \quad r s(r-s)(x-y) \neq 0 ; \\
& E(r, 0 ; x, y)=E(0, r ; x, y)=L_{r}(x, y)=\left(L\left(x^{r}, y^{r}\right)\right)^{1 / r}, \quad r(x-y) \neq 0 ; \\
& E(r, r ; x, y)=I_{r}(x, y)=\left(I\left(x^{r}, y^{r}\right)\right)^{1 / r}, \quad x-y \neq 0 ; \\
& E(0,0 ; x, y)=G(x, y), \quad x \neq y ; \\
& E(r, s ; x, x)=x, \quad x=y .
\end{aligned}
$$

He showed that $E$ can be extended to be continuous on the domain

$$
\{(r, s ; x, y): r, s \in R, x, y>0\} .
$$

A function similar to $E$ that involves a transformation of values of $(r, s)$ was given by Cisbani [4] and by Tobey [23]. 
Toader in $[21,22]$ considered general means

$$
M_{r, s}(x, y)=\left(C_{r s} \cdot \frac{f_{r}(x, y)}{g_{s}(x, y)}\right)^{1 /(r-s)},
$$

where $f_{r}$ and $g_{s}$ are homogeneous functions of degree $r$ and $s$, respectively, and $C_{r s}=$ $\lim _{t \rightarrow 1}\left(g_{s}(1, t) / f_{r}(1, t)\right)$.

The author also researched the mean values in $[16,18]$ and the extended means $E$ in $[17,19]$ by a simpler method.

It is easy to see that these particular means above are special cases of means introduced by Tobey [23]. The study of these means has a rich literature, e.g., for details see $[3,8,9,10,11,12,13,14,16,17]$.

We introduce below a more complicated two-parameter family of nonhomogeneous means $E_{2 n}(r, s ; x, y)$, and give their basic properties and basic results concerning monotonicity, comparability, and the like.

Study of $E_{2 n}$ is interesting, both because most of the two-variable means stated above are special cases of $E_{2 n}$, and because it is challenging to study a function whose formulation is so indeterminate.

\section{Definitions and Basic Properties}

Let $U_{n}(t ; u)$ be a sequence satisfying

$$
t \partial U_{n}(t ; u) / \partial t-(n+1) U_{n}(t ; u)=U_{n+1}(t ; u),
$$

and $U_{0}(t ; u)=u^{t}$ for $u>0, n \in N$.

Definition 1. The extended logarithmic means of $2 n$-th order $L_{2 n}(r ; x, y)$ are a one-parameter family of nonhomogeneous means defined, for $n \in N$, as

$$
\begin{aligned}
& L_{2 n}(r ; x, y)=\left[\frac{2 n+1}{r^{2 n+1}} \cdot \frac{U_{2 n}(r ; y)-U_{2 n}(r ; x)}{(\ln y)^{2 n+1}-(\ln x)^{2 n+1}}\right]^{1 / r}, \quad r(x-y) \neq 0 ; \\
& L_{2 n}(0 ; x, y)=\exp \left[\frac{2 n+1}{2 n+2} \cdot \frac{\left.(\ln y)^{2 n+2}-(\ln x)^{2 n+2}\right\urcorner}{(\ln y)^{2 n+1}-(\ln x)^{2 n+1}}, \quad x \neq y ;\right. \\
& L_{2 n}(r ; x, x)=x, \quad r \in R .
\end{aligned}
$$

Definition 2. The extended means of $2 n$-th order $E_{2 n}(r, s ; x, y)$ are a two-parameter family of nonhomogeneous means defined, for $n \in N$, by

$$
\begin{array}{ll}
E_{2 n}(r, s ; x, y)=\left[\frac{r^{2 n+1}}{s^{2 n+1}} \cdot \frac{U_{2 n}(s ; y)-U_{2 n}(s ; x)}{U_{2 n}(r ; y)-U_{2 n}(r ; x)}\right]^{1 /(s-r)}, \quad r s(s-r)(x-y) \neq 0 \\
E_{2 n}(r, r ; x, y)=\exp \left[\frac{1}{r} \cdot \frac{U_{2 n+1}(r ; y)-U_{2 n+1}(r ; x)}{U_{2 n}(r ; y)-U_{2 n}(r ; x)}\right], \quad r(x-y) \neq 0 \\
E_{2 n}(r, 0 ; x, y)=L_{2 n}(r ; x, y), \quad x \neq y, r \in R \\
E_{2 n}(r, s ; x, x)=x, \quad r, s \in R .
\end{array}
$$


For the sake of convenience, we write $E_{2 n}(r, s ; x, y)=E_{2 n}(r, s)=E_{2 n}(x, y)=E_{2 n}$, shifting notation to suit the context.

Theorem. 1. $L_{2 n}(r ; x, y)$ and $E_{2 n}(r, s ; x, y)$ can be expressed in integral forms:

$$
\begin{aligned}
L_{2 n}(r ; x, y) & =E_{2 n}(r, 0 ; x, y)=\left[\frac{\int_{x}^{y}(\ln u)^{2 n} u^{r-1} d u}{\int_{x}^{y}(\ln u)^{2 n} u^{-1} d u}\right]^{1 / r}, \quad r(x-y) \neq 0 ; \\
L_{2 n}(0 ; x, y) & =\exp \left(\frac{\int_{x}^{y}(\ln u)^{2 n+1} u^{-1} d u}{\int_{x}^{y}(\ln u)^{2 n} u^{-1} d u}\right), \quad x \neq y ; \\
E_{2 n}(r, s ; x, y) & =\left(\frac{\int_{x}^{y}(\ln u)^{2 n} u^{s-1} d u}{\int_{x}^{y}(\ln u)^{2 n} u^{r-1} d u}\right)^{1 /(s-r)}, \quad(r-s)(x-y) \neq 0 ; \\
E_{2 n}(r, r ; x, y) & =\exp \left(\frac{\int_{x}^{y}(\ln u)^{2 n+1} u^{r-1} d u}{\int_{x}^{y}(\ln u)^{2 n} u^{r-1} d u}\right), \quad x \neq y .
\end{aligned}
$$

Proof. Let $g(t ; x, y)=\left(y^{t}-x^{t}\right) / t$ for $t \neq 0$ and $g(0 ; x, y)=\ln y-\ln x$. By direct computation and induction on $n$, it follows that

$$
\begin{aligned}
g_{t}^{(n)}(t ; x, y) & =\left(U_{n}(t ; y)-U_{n}(t ; x)\right) / t^{n+1}, \\
\partial U_{n}(t ; u) / \partial u & =(\ln u)^{n} u^{t-1} t^{n+1}, \\
g_{t}^{(n)}(t ; x, y) & =\int_{x}^{y}(\ln u)^{n} u^{t-1} d u .
\end{aligned}
$$

This implies Theorem 1 .

Corollary 1. $L_{2 n}(r ; x, y)$ is continuous on the domain

$$
\{(r ; x, y) \mid x>0, y>0, r \in R\}
$$

and $E_{2 n}(r, s ; x, y)$ is continuous on the domain

$$
\{(r, s ; x, y) \mid x>0, y>0, r, s \in R\} .
$$

Theorem 2. $L_{2 n}(r ; x, y)$ and $E_{2 n}(r, s ; x, y)$ have the following properties:

$$
\begin{aligned}
& \min \{x, y\} \leq L_{2 n}(r ; x, y) \leq \max \{x, y\}, \\
& L_{2 n}(r ; x, y)=L_{2 n}(r ; y, x), \\
& L_{0}(r ; x, y)=L_{r}(x, y), \\
& L_{2 n}\left(r ; x^{\alpha}, y^{\alpha}\right)=\left(L_{2 n}(\alpha r ; x, y)\right)^{\alpha}, \quad \alpha \neq 0 ; \\
& \min \{x, y\} \leq E_{2 n}(r, s ; x, y) \leq \max \{x, y\}, \\
& E_{2 n}(r, s ; x, y)=E_{2 n}(r, s ; y, x)=E_{2 n}(s, r ; x, y), \\
& E_{0}(r, s ; x, y)=E(r, s ; x, y), \\
& E_{0}(r, r ; x, y)=I_{r}(x, y), \\
& {\left[E_{2 n}(r, t)\right]^{r-t}=\left[E_{2 n}(r, s)\right]^{r-s}\left[E_{2 n}(s, t)\right]^{s-t},} \\
& {\left[E_{2 n}(\alpha r, \alpha s ; x, y)\right]^{\alpha}=E_{2 n}\left(r, s ; x^{\alpha}, y^{\alpha}\right), \quad \alpha \neq 0 .}
\end{aligned}
$$


Proof. They follow from the mean value theorem for integral, Theorem 1 and standard arguments.

Lemma 1. Let $f, h:[a, b] \rightarrow R$ be integrable functions, both increasing or both decreasing. Furthermore, let $p:[a, b] \rightarrow R^{+}$be an integrable function. Then

$$
\int_{a}^{b} p(u) f(u) d u \int_{a}^{b} p(u) h(u) d u \leq \int_{a}^{b} p(u) d u \int_{a}^{b} p(u) f(u) h(u) d u .
$$

If one of the functions of $f$ or $h$ is nonincreasing and the other nondecreasing, then the inequality (21) reverses.

Inequality (21) is called the Tchebycheff integral inequality. For proof of it, see $[2,7$, $8,12,13,18]$.

Proposition 1. Let $g=g(t ; x, y)=\int_{x}^{y} u^{t-1} d u, x \neq y$. Then, for $k, j \in N$, we have

$$
g_{t}^{(2 k+1)} g_{t}^{(2(j+k)+1)} \leq g_{t}^{(2 k)} g_{t}^{(2(j+k+1))}
$$

The ratio $g_{t}^{(2(j+k)+1)}(t ; x, y) / g_{t}^{(2 k)}(t ; x, y)$ is increasing in $t$.

Proof. Inequality (22) is a special case of the Tchebycheff (or Čebyšev) integral inequality applied to the functions $p(u)=(\ln u)^{2 k} u^{t-1}, f(u)=\ln u$ and $h(u)=(\ln u)^{2 j+1}$ for $j, k \in N, t \in R$ and $u \in[x, y]$.

Inequality (22) and direct calculation produce

$$
\left(\frac{g_{t}^{(2(j+k)+1)}}{g_{t}^{(2 k)}}\right)_{t}=\frac{g_{t}^{(2(j+k+1))} g_{t}^{(2 k)}-g_{t}^{(2(j+k)+1)} g_{t}^{(2 k+1)}}{\left(g_{t}^{(2 k)}\right)^{2}} \geq 0 .
$$

Therefore, the desired result follows.

Theorem 3. $L_{2 n}(r ; x, y)$ increases with respect to $r$.

Proof. From Theorem 1 we have

$$
\begin{aligned}
{\left[\ln L_{2 n}(r ; x, y)\right]_{t} } & =\frac{1}{r^{2}}\left(\frac{\int_{x}^{y}(\ln u)^{2 n+1} u^{r-1} d u}{\int_{x}^{y}(\ln u)^{2 n} u^{r-1} d u} \cdot r-\ln \frac{\int_{x}^{y}(\ln u)^{2 n} u^{r-1} d u}{\int_{x}^{y}(\ln u)^{2 n} u^{-1} d u}\right) \\
& =\frac{1}{r}\left(\frac{\int_{x}^{y}(\ln u)^{2 n+1} u^{r-1} d u}{\int_{x}^{y}(\ln u)^{2 n} u^{r-1} d u}-\frac{\int_{x}^{y}(\ln u)^{2 n+1} u^{\theta-1} d u}{\int_{x}^{y}(\ln u)^{2 n} u^{\theta-1} d u}\right)
\end{aligned}
$$

where $\theta$ is between 0 and $r$, by the mean value theorem for derivative. From Proposition 1 , $\left[\ln L_{2 n}(r ; x, y)\right]_{t}>0$, therefore $L_{2 n}(r ; x, y)$ is increasing with respect to $r$ for $n \in N$.

Theorem 4. If $x, y>1$, then

$$
L_{2 n}(r ; x, y)=E_{2 n}(r, 0 ; x, y) \leq E_{2 n+2}(r, 0 ; x, y)=L_{2 n+2}(r ; x, y) .
$$

If $0<x<y<1$, inequality (23) is reversed. 
Proof. Using formulae (14) and (15), the Tchebycheff integral inequality applied to $p(u)=u^{-1}(\ln u)^{2 n}, f(u)=u^{r}$ and $h(u)=(\ln u)^{2}$, and the standard arguments result in Theorem 4.

Theorem 5. $L_{2 n}(r ; x, y)$ increases in both $x$ and $y$.

Proof. From (15) and (18), direct computation produces

$$
\begin{aligned}
\ln L_{2 n}(0 ; x, y)= & g_{r}^{(2 n+1)}(0 ; x, y) / g_{r}^{(2 n)}(0 ; x, y), \\
\frac{\partial\left[\ln L_{2 n}(0 ; x, y)\right]}{\partial x}= & {\left[\int_{x}^{y}(\ln u)^{2 n+1} u^{-1} d u\right.} \\
& \left.-(\ln x) \int_{x}^{y}(\ln u)^{2 n} u^{-1} d u\right] \frac{(\ln x)^{2 n} x^{-1}}{\left[g_{r}^{(2 n)}(0 ; x, y)\right]^{2}} \geq 0 .
\end{aligned}
$$

Hence, $L_{2 n}(0 ; x, y)$ increases with respect to both $x$ and $y$.

Since $L_{2 n}^{r}(r ; x, y)=g_{r}^{(2 n)}(r ; x, y) / g_{r}^{(2 n)}(0 ; x, y)$, easy calculation results in

$$
\begin{aligned}
\frac{\partial L_{2 n}^{r}(r ; x, y)}{\partial x} & =\left[g_{r}^{(2 n)}(r ; x, y)-x^{r} g_{r}^{(2 n)}(0 ; x, y)\right] x^{-1}(\ln x)^{2 n} /\left[g_{r}^{(2 n)}(0 ; x, y)\right]^{2} \\
& =\left[\int_{x}^{y}(\ln u)^{2 n} u^{r-1} d u-x^{r} \int_{x}^{y}(\ln u)^{2 n} u^{-1} d u\right] \frac{(\ln x)^{2 n} x^{-1}}{\left[g_{r}^{(2 n)}(0 ; x, y)\right]^{2}}, \\
\operatorname{sgn}\left(\partial L_{2 n}^{r}(r ; x, y) / \partial x\right) & =\operatorname{sgn} r .
\end{aligned}
$$

Therefore, $L_{2 n}(r ; x, y)$ increases with both $x$ and $y$.

\section{Monotonicity of $E_{2 n}(r, s ; x, y)$}

Theorem 6. $E_{2 n}(r, s ; x, y)$ increases with respect to both $r$ and $s$.

Proof. From (17) and Proposition 1, it follows that $E_{2 n}(r, r ; x, y)$ increases with both $r$ and $s$.

By easy computation, we have

$$
\left[\ln E_{2 n}(r, s ; x, y)\right]_{s}=\frac{1}{(s-r)^{2}}\left[\frac{g_{s}^{2 n+1}(s ; x, y)}{g_{s}^{(2 n)}(s ; x, y)}(s-r)-\ln \frac{g_{s}^{(2 n)}(s ; x, y)}{g_{r}^{(2 n)}(r ; x, y)}\right] .
$$

By the mean value theorem, we obtain

$$
\ln \frac{g_{s}^{(2 n)}(s ; x, y)}{g_{r}^{(2 n)}(r ; x, y)}=\frac{g_{\gamma}^{(2 n+1)}(\gamma ; x, y)}{g_{\gamma}^{(2 n)}(\gamma ; x, y)}(s-r)<\frac{g_{s}^{(2 n+1)}(s ; x, y)}{g_{s}^{(2 n)}(s ; x, y)}(s-r)
$$

where $\gamma$ is between $r$ and $s$. Therefore, $\left[\ln E_{2 n}(r, s ; x, y)\right]_{s}>0, E_{2 n}(r, s ; x, y)$ is increasing in $s$. Since $E_{2 n}(r, s ; x, y)=E_{2 n}(s, r ; x, y)$, it is deduced that $E_{2 n}(r, s ; x, y)$ increases in both $r$ and $s$. 
Theorem 7. For $x, y>1$,

$$
E_{2 n}(r, s ; x, y) \leq E_{2 n+2}(r, s ; x, y) .
$$

If $0<x<y<1$, inequality (24) reverses.

Proof. For $s>r$, inequality (24) is equivalent to

$$
\frac{g_{s}^{(2 n+2)}(s ; x, y)}{g_{r}^{(2 n+2)}(r ; x, y)} \geq \frac{g_{s}^{(2 n)}(s ; x, y)}{g_{r}^{(2 n)}(r ; x, y)},
$$

that is

$$
\frac{\int_{x}^{y}(\ln u)^{2 n+2} u^{s-1} d u}{\int_{x}^{y}(\ln u)^{2 n+2} u^{r-1} d u} \geq \frac{\int_{x}^{y}(\ln u)^{2 n} u^{s-1} d u}{\int_{x}^{y}(\ln u)^{2 n} u^{r-1} d u} .
$$

From $(21)$, applied to $p(u)=(\ln u)^{2 n} u^{r-1}, f(u)=(\ln u)^{2}, g(u)=u^{s-r}$, the inequality (24) follows.

Theorem 8. $E_{2 n}(r, s ; x, y)$ increases with both $x$ and $y$.

Proof. Since $\ln E_{2 n}(r, r ; x, y)=g_{r}^{(2 n+1)}(r ; x, y) / g_{r}^{(2 n)}(r ; x, y)$, calculating straightforwardly yields

$$
\begin{aligned}
& {\left[\ln E_{2 n}(r, r ; x, y)\right]_{x}=\left[g_{r}^{(2 n+1)}(r ; x, y)-(\ln x) g_{r}^{(2 n)}(r ; x, y)\right] \frac{x^{r-1}(\ln x)^{2 n}}{\left[g_{r}^{(2 n)}(r ; x, y)\right]^{2}}} \\
& =\left[\int_{x}^{y}(\ln u)^{2 n+1} u^{r-1} d u-(\ln x) \int_{x}^{y}(\ln u)^{2 n} u^{r-1} d u\right] \frac{x^{r-1}(\ln x)^{2 n}}{\left[g_{r}^{(2 n)}(r ; x, y)\right]^{2}} \geq 0 .
\end{aligned}
$$

Thus, $E_{2 n}(r, r ; x, y)$ is increasing in both $x$ and $y$.

Let

$$
P_{2 n}(r, s ; x, y)=\left[E_{2 n}(r, s ; x, y)\right]^{s-r} \cdot \frac{s^{2 n+1}}{r^{2 n+1}}=\frac{U_{2 n}(s ; y)-U_{2 n}(s ; x)}{U_{2 n}(r ; y)-U_{2 n}(r ; x)} .
$$

Computating directly arrives at

$$
\begin{aligned}
\frac{\partial P_{2 n}}{\partial x} & =\frac{(\ln x)^{2 n}(r s)^{2 n+1} x^{r+s-1}}{\left[U_{2 n}(r ; y)-U_{2 n}(r ; x)\right]^{2}}\left[\frac{g_{r}^{(2 n)}(r ; y, x)}{x^{r}}-\frac{g_{s}^{(2 n)}(s ; y, x)}{x^{s}}\right] \\
\left(\frac{g_{t}^{(2 n)}(t ; y, x)}{x^{t}}\right)_{t} & =\frac{g_{t}^{(2 n+1)}(t ; y, x)-(\ln x) g_{t}^{(2 n)}(t ; y, x)}{x^{t}} \\
& =\frac{\int_{y}^{x}(\ln u)^{2 n+1} u^{t-1} d u-(\ln x) \int_{y}^{x}(\ln u)^{2 n} u^{t-1} d u}{x^{t}}
\end{aligned}
$$

From this we conclude that

$$
\begin{aligned}
\operatorname{sgn}\left(\frac{\partial E_{2 n}^{s-r}}{\partial x}\right) & =\operatorname{sgn}\left(\frac{r^{2 n+1}}{s^{2 n+1}} \cdot \frac{\partial P_{2 n}}{\partial x}\right) \\
& =\operatorname{sgn}\left(\frac{g_{r}^{(2 n)}(r ; y, x)}{x^{r}}-\frac{g_{s}^{(2 n)}(s ; y, x)}{x^{s}}\right)=\operatorname{sgn}(s-r) .
\end{aligned}
$$


Therefore, $\partial E_{2 n}(r, s ; x, y) / \partial x>0, E_{2 n}(r, s ; x, y)$ is increasing with both $x$ and $y$. $19]$.

Remark. Some properties of the function $g(t ; x, y)$ had been given in $[16,17,18$,

\section{Acknowledgements}

The author is indebted to the referee for many helpful suggestions and for many corrections.

\section{References}

[1] Horst Alzer, "On Stolarsky's mean value family," Internat. J. Math. Educat. Sci. Tech., 20(1)(1989), 186-189.

[2] E. F. Beckenbach and R. Bellman, Inequalities, Springer-Verlag, Berlin, 1961.

[3] B. C. Carlson, "The logarithmic mean," Amer. Math. Monthly, 79(1972), 615-618.

[4] R. Cisbani, "Contributi alla teoria delle medie I," Metron, 13(2)(1938), 23-34.

[5] Ji Chen and Hai-bing Shu, "Refinements of Ostile-Terwillinger's inequality," Shuxue Tongxun, (1988), no. 3, 7-8, (in Chinese).

[6] L. Galvani, "Dei limiti a cui tendono alcune media," Boll. Un. Mat. Ital., (6)1927, 173-179.

[7] G. H. Hardy, J. E. Littlewood and G. Polya, Inequalities, 2nd Edition, Cambridge University Press, Cambridge, 1959.

[8] Ji-chang Kuang, Applied Inequalities, 2nd Edition, Hunan Education Press, Changsha, China, 1993, (in Chinese).

[9] E. Leach and M. Sholander, "Extended mean values," Amer. Math. Monthly, 85(1978), 84-90.

[10] E. Leach and M. Sholander. "Extended mean values II," J. Math. Anal. Appl., 92(1983), 207-223.

[11] Tung-Po Lin, "The power mean and the logarithmic mean," Amer. Math. Monthly, 81(1974), 879-883.

[12] D. S. Mitrinović, Analytic Inequalities, Springer-Verlag, Berlin, 1970.

[13] D. S. Mitrinović, J. E. Pecaric and A. M. Fink, Classical and New Inequalities and Analysis, Kluwer Academic Publishers, Dordrecht, 1993.

[14] Z. Páles, "Inequalities for differences of powers," J. Math. Anal. Appl. 131(1988), 271-281.

[15] G. Pólya and G. Szegö, Isoperimetric Inequalities in Mathematical Physics, Princeton University Press, Princeton, 1951.

[16] Feng QI and Qiu-ming Luo, "Refinements and extensions of an inequality," Mathematics and Informatics Quarterly, 1998 (to appear).

[17] Feng QI and Qiu-ming Luo, "A simple proof of monotonicity for extended means values," J. Math. Anal. Appl., 1998, (to appear).

[18] Feng QI and Shn-lin Xu, "Refinements and extensions of an inequality II," Journal of Mathematical Analysis and Applications, 221(1997), 616-620.

[19] Feng QI and Sen-lin Xu, "The function $\left(b^{x}-a^{x}\right) / x$ : Inequalities and properties," Proceedings of American the Mathematical Society, 1998 (to appear).

[20] K. B. Stolarsky, "Generalizations of the logarithmic mean," Math. Mag., 48(1975), 87-92. 
[21] Gh. Toader, First Conf. Appl. Math. Mech., Cluj-Napoca, 1988.

[22] Gh. Toader, "A generalization of geometric (or) harmonic means," 'Babes-Bolyai' Univ. Fac. Math. Phys. Res. Sem. Math. Anal. (2)1989, 21-28.

[23] M. D. Tobey, "A two-parameter homogeneous mean value," Proc. Amer. Math. Soc., 18(1967), 9-14.

[24] Ren-er Yang and Dong-ji Cao, "Generalizations of the logarithmic mean," J. Ningbo Univ. (2) 1989, 105-108.

[25] Feng QI, "Generalized weighted mean values with two parameters," Proceedings of the Royal Society of London A 454(1998), 1-10.

Department of Mathematics, Jiaozuo Institute of Technology, Jiaozuo 454000, Henan, The People's Republic of China.

Department of Mathematics, University of Science and Technology of China, Hefei 230026, Anhui, The People's Republic of China. 